\title{
Use of nonlinear regression and nonlinear mathematical programming in the formulation of substrate mixtures for soil-less culture - a review -
}

\author{
J.A. Díaz-García ${ }^{1 *}$ and R. Requejo-López ${ }^{2}$ \\ ${ }^{1}$ Universidad Autónoma Agraria Antonio Narro, Department of Statistics and Computation, 25315 Buenavista, \\ Saltillo, Coahuila, México. ${ }^{2}$ Universidad Autónoma Agraria Antonio Narro, Department of Soil Science, \\ 25315 Buenavista, Saltillo, Coahuila, México. "Corresponding author: jadiaz@uaaan.mx
}

\begin{abstract}
With respect to four inorganic and organic substrates - tezontle (volcanic rock), perlite, peat and coir - we studied the relevant physical and chemical properties of mixtures of two of these substrates. A nonlinear regression model was used to analyse the physical properties of each possible mixture. Finally, a methodology is proposed for formulating mixtures of substrates as a deterministic nonlinear mathematical programming problem.
\end{abstract}

Keywords: Coir; Peat; Nonlinear regression, Nonlinear optimisation; Substrate mixtures; Tezontle. 


\section{Introduction}

Soil serves as a support for plants and is the medium from which they obtain water, mineral substances and oxygen, which are essential for growth and vegetative development. An ideal soil has a porosity and particle disposition that enables root penetration while retaining appropriate amounts of water and air [Foth and Ellis (1988), Hablin et al. (1999), and Russell (2008)].

However, such soils may not be available, and then artificial soils may be used. This is the case of most flowerpot and containerised crops. Such plants have limited root growth, but they still need nutrients, air and water. For this reason, artificial substrates are sometimes required. These are often obtained by mixtures of various products, and the combination of the characteristics of each component provides the overall (and hopefully, optimal) characteristics of the substrate [Resh (2002a), Resh (2002b) and Russell (2008)].

The apparent density of a substrate should be low, so that the roots can penetrate it easily, and at the same time, to minimise the weight of the containerised crops. Organic matter has high porosity, ionic interchange and water retention capabilities, together with low density. All these properties are recommended for a substrate, which explains why most artificial substrates are essentially composed of organic matter; the remaining components are usually constituted of natural minerals or of artificial minerals such as tezontle, sand, perlite or vermiculite. These mineral products are very porous, with a high real density and a very low apparent density. In general, the grain size of an artificial substrate is higher than that of the soil, which facilitates air infiltration; at the same time, however, this property reduces water retention. This consideration explains why mixtures are usually composed of both organic and mineral substances, to achieve an equilibrium between air infiltration and water retention [Resh (2002a), Resh (2002b) and Russell (2008)].
The chemical properties of a substrate are important because they are the main factors responsible for the availability of nutrients. However, new crops are increasingly being constituted in such a way that the physical properties of a substrate prevail as its desired characteristics, with all the additional plant necessities being provided by nutritive substances, which are permanently available, being recycled to the plant by the irrigation system [Hablin et al. (1999), Resh (2002b) and Russell (2008)].

In many countries, substrates are still obtained by traditional trial and error methods; mixtures are formulated by arbitrarily establishing the proportions of the respective components, and the resulting mixture is then accepted if it improves the overall crop yield. The resulting mixture is not necessarily the optimal one, as the method does not explore all the possible combinations of the available components. The time employed in evaluating the mixtures, the cost of the materials used and the search for optimal resources are among the aspects contributing to the complexity of substrate formulation, given the number of variables involved in the problem (Zamora Morales et al. 2005).

Linear programming is a commonly-used empirical technique for formulating substrate mixtures used in a soli-less culture, and generally provides good approximations (Burés et al. (1988), Burés (1997) and Zamora Morales et al. (2005), among many others). However, most authors accept that the constraints imposed in the linear programming are not completely suitable, because they are established over design variables which do not present a linear behaviour.

This paper proposes a general methodology, based on nonlinear regression models and nonlinear mathematical programming, for obtaining substrate 
mixtures for soil-less culture comprised of two inorganic or organic substrates, to be used for protected and containerised crops. For this purpose, we study the physical and chemical properties of four inorganic and organic substrates - tezontle (volcanic rock), perlite, peat and coir - together with those of the mixtures comprising different proportions of two of these four substrates, see Section 2.

Subsequently, the physical properties of the mixtures are modelled by nonlinear regression models, according to the respective substrate proportions in each mixture, see Section 3. These models are then used to replace the linear restrictions corresponding to the physical properties expressed in the linear mathematical programming problem; thus, we obtain a nonlinear mathematical programming solution. The corresponding linear and nonlinear problems are solved, compared and analysed using the commercial software LINGO.

\section{Physical and chemical properties of substrates and mixtures}

Four substrates were used in the mixture formulation tests: perlite, tezontle, peat and coir. Following the methodology proposed by Pire and Pereira (2003), Gabriel et al. (1993) and North Carolina State University (2007), we determined, in triple analysis, the following physical and chemical properties: total porous space (TPS), air porosity (AP), humidity retention (HR), apparent density (AD), real density (RD), ashes(A), organic matter (OM), $\mathrm{pH}$ and electric conductivity (EC), which are shown in Table 1.

We also formulated six mixtures (denoted by a $M_{1}-M_{6}$ and defined in Tables 2 and 3) composed of two substrates with eleven different proportions. Using the above-mentioned procedure, their physical and chemical properties to be used in the formulation of mixtures of substrates were determined (Burés et al., 1988); see Tables 2 and 3.

Table 1. Physical and chemical properties of each substrate

\begin{tabular}{lccccccccc}
\hline Substrate & TPS & AP & HR & AD & RD & A & OM & $\mathrm{pH}$ & EC \\
\hline Perlite & $\%$ & $\%$ & $\%$ & $\mathrm{gcc}^{-1}$ & $\mathrm{gcc}^{-1}$ & $\%$ & $\%$ & & $\mathrm{dSm}^{-1}$ \\
Tezontle & 52.40 & 5.43 & 56.97 & 0.19 & 0.51 & 100 & 0 & 7.6 & 1.3 \\
Peat & 72.65 & 8.38 & 64.27 & 0.07 & 0.28 & 10 & 90 & 6.4 & 1.2 \\
Coir & 81.83 & 4.48 & 77.35 & 0.09 & 0.49 & 10 & 90 & 7.2 & 3.7 \\
\hline
\end{tabular}

Table 2. Physical and chemical properties of the mixtures

\begin{tabular}{cccccccccc}
\hline & \multicolumn{3}{c}{$\mathrm{M}_{1}=$ Perlite + Coir } & \multicolumn{3}{c}{$\mathrm{M}_{2}=$ Perlite + peat } & \multicolumn{3}{c}{$\mathrm{M}_{3}=$ Perlite + Tezontle } \\
\hline & TPS & HR & OM & TPS & HR & OM & TPS & HR & OM \\
\hline & $\%$ & $\%$ & $\%$ & $\%$ & $\%$ & $\%$ & $\%$ & $\%$ & $\%$ \\
\hline $0 \%+100 \%$ & 81.83 & 77.35 & 90 & 72.65 & 64.27 & 90 & 50.70 & 42.89 & 0 \\
$10 \%+90 \%$ & 84.77 & 81.06 & 70 & 77.23 & 66.66 & 90 & 51.77 & 47.20 & 0 \\
$20 \%+80 \%$ & 85.34 & 79.34 & 50 & 85.17 & 75.46 & 70 & 54.17 & 50.46 & 0 \\
$30 \%+70 \%$ & 82.86 & 77.71 & 40 & 84.80 & 74.51 & 50 & 51.91 & 50.20 & 0 \\
\hline
\end{tabular}


Continued...

\begin{tabular}{cccccccccc}
\hline & \multicolumn{3}{c}{$\mathrm{M}_{1}=$ Perlite + Coir } & \multicolumn{3}{c}{$\mathrm{M}_{2}=$ Perlite + peat } & \multicolumn{3}{c}{$\mathrm{M}_{3}=$ Perlite + Tezontle } \\
\hline & TPS & HR & OM & TPS & HR & OM & TPS & HR & OM \\
\hline & $\%$ & $\%$ & $\%$ & $\%$ & $\%$ & $\%$ & $\%$ & $\%$ & $\%$ \\
\hline $40 \%+60 \%$ & 76.06 & 71.49 & 30 & 82.77 & 75.63 & 60 & 50.31 & 48.60 & 0 \\
$50 \%+50 \%$ & 73.29 & 69.86 & 30 & 79.60 & 75.60 & 40 & 51.80 & 49.80 & 0 \\
$60 \%+40 \%$ & 72.69 & 69.83 & 20 & 79.00 & 73.86 & 40 & 55.54 & 53.26 & 0 \\
$70 \%+30 \%$ & 67.20 & 63.20 & 20 & 71.20 & 68.06 & 20 & 58.43 & 53.29 & 0 \\
$80 \%+20 \%$ & 64.83 & 63.40 & 10 & 67.51 & 64.66 & 20 & 57.89 & 54.74 & 0 \\
$90 \%+10 \%$ & 66.11 & 64.11 & 0 & 63.49 & 60.63 & 10 & 59.89 & 57.03 & 0 \\
$100 \%+0 \%$ & 62.40 & 56.97 & 0 & 62.40 & 56.97 & 0 & 62.40 & 56.97 & 0 \\
\hline
\end{tabular}

Table 3. Physical and chemical properties

\begin{tabular}{cccccccccc}
\hline & \multicolumn{3}{c}{$\mathrm{M}_{4}=$ peat + Tezontle } & \multicolumn{3}{c}{$\mathrm{M}_{5}=$ Coir + Tezontle } & \multicolumn{3}{c}{$\mathrm{M}_{6}=$ peat + Coir } \\
\hline & TPS & HR & OM & TPS & HR & OM & TPS & HR & OM \\
\hline 0 & $\%$ & $\%$ & $\%$ & $\%$ & $\%$ & $\%$ & $\%$ & $\%$ \\
\hline $0 \%+100 \%$ & 50.7 & 42.89 & 0 & 50.7 & 42.89 & 0 & 81.83 & 77.35 & 90 \\
$10 \%+90 \%$ & 51.23 & 49.8 & 0 & 48.43 & 46.71 & 10 & 83.74 & 76.89 & 90 \\
$20 \%+80 \%$ & 53.45 & 51.74 & 0 & 51.89 & 50.17 & 10 & 82.46 & 76.17 & 90 \\
$30 \%+70 \%$ & 52.91 & 50.91 & 0 & 54.77 & 53.34 & 10 & 65.23 & 57.51 & 90 \\
$40 \%+60 \%$ & 58.99 & 56.71 & 10 & 59.29 & 57.86 & 10 & 65.31 & 59.03 & 90 \\
$50 \%+50 \%$ & 61.39 & 59.39 & 10 & 62.29 & 60.86 & 10 & 75.89 & 68.46 & 90 \\
$60 \%+40 \%$ & 65.45 & 62.88 & 0 & 64.2 & 62.2 & 20 & 60.43 & 51.86 & 90 \\
$70 \%+30 \%$ & 66.57 & 62.85 & 20 & 69,17 & 67,17 & 20 & 63.54 & 56.4 & 90 \\
$80 \%+20 \%$ & 67.17 & 62.88 & 20 & 72.8 & 70.23 & 20 & 77.94 & 63.09 & 90 \\
$90 \%+10 \%$ & 73.62 & 67.62 & 30 & 76.8 & 73.66 & 30 & 74.11 & 51.54 & 90 \\
$100 \%+0 \%$ & 72.65 & 64.27 & 90 & 81.83 & 77.35 & 90 & 72.65 & 64.27 & 90 \\
\hline
\end{tabular}

\section{Modelling the physical and chemical properties of the mixtures}

As remarked above, in general the physical and chemical properties of substrates (and their mixtures) do not present a linear behaviour. In this section, we study the functional behaviour of the physical properties TPS, HR and OM in the mixtures $\mathrm{M}^{1}-\mathrm{M}_{6}{ }^{1}$.

1 The interest in this particular modelling is detailed in Section 4.
The results were obtained by the implementation of a program in S-PLUS (Lam (2011)) to fit the different nonlinear regression models. The least squares method was applied to the following fitting models:

- Linear regression model

$$
Y=p_{1} x_{1}+p_{2} x_{2}+\mathrm{x}
$$


- Polynomial regression model

$$
\begin{aligned}
& Y=p_{1} x_{1}+p_{2} x_{2}+p_{3} x_{1}^{2}+p_{4} x_{2}^{2}+\cdots \\
& +p_{k} x_{1}^{k}+p_{k+1} x_{2}^{k}+p_{k+2} x_{1} x_{2}+\xi
\end{aligned}
$$

- Reciprocal regression model

$$
Y=\frac{\text { polynomial1 }}{\text { Polynomial2 }}+\xi
$$

- Logarithmic regression model

$$
Y=p_{1} \log x_{1}+p_{2} \log x_{2}+\xi
$$

- Exponential regression model

$Y=p_{1} \exp \left\{p_{2} x_{1}+p_{3} x_{2}+p_{4} x_{1}^{2}+p_{5} x_{1}^{3}\right\}+\xi$

The best model was selected by means of the minimum mean square error criterion (Graybill and Iyer (1994)).

In general, the models fitted take the form

$$
Y=f\left(x_{1}, x_{2}\right)+\xi
$$

where $Y$ is the mixture variable to be modelled (TPS, HR or OM); $f\left(x_{1}, x_{2}\right)$ is the functional form of the model to be fitted; $x_{1}, x_{2}$ denote the proportions of two of the substrates(selected from perlite, tezontle, peat and coir) making up the mixture, where $0 \leq x_{k} \leq 1, k=1,2$, and $\xi$ is the error. Note also that $x_{1}, x_{2}$ satisfy $x_{1}+x_{2}=1$ which means that $x_{1}$ and $x_{2}$ are linearly dependent, moreover, $x_{1}=1-x_{2}$ or $x_{2}=1-x_{1}$. A priori, all that is necessay is to fit a model in terms of $x_{1}$ (or $x_{2}$ ). However, in this study we wish to obtain a mathematical model for computing the physical property $Y$ of the mixture, when the proportions and the individual physical properties of the corresponding component of the substrate are known. This model is then used in the nonlinear optimisation program formulation. Strictly speaking, we do not estimate the model for any possible value of $x_{1}$ and $x_{2}$, but rather, in the subspace defined by the line $x_{1}+x_{2}=1$. A possible drawback is the fact that few experimental points (eleven) are used for fitting the TPS, HR and OM characteristic models (see Tables 2-3). Fortunately, the experimental data present a smooth behaviour, and, most importantly, they provide information over the whole domain of $x_{1}$ and $x_{2}$ (specifically, the domain is the line $x_{1}+x_{2}=1$ in the first octant of $\mathrm{R}^{3}$ ). Moreover, it is important to take into account that models of the form $Y=p_{0}+p_{1} z_{1}+p_{2} z_{2}+\xi$ (with $p_{0} \neq 0$ ), or similar should not be considered, because when $x_{1}=x_{2}=0$ we obtain $Y=p_{0}$, but the correct result is $Y=0$. Finally, the most complicated aspect of this study is to propose the same functional form for each characteristic (TPS, HR and OM) and for all the possible mixtures obtained.

As shown in Tables 2-3, the variable OM presents a linear behaviour. Then, we just need to determine the behaviour of TPS and HR. In both cases, a well fitted model for TPS and HR for the six mixtures has the form

$Y=p_{1} z_{1}+p_{2} z_{2} \exp \left\{p_{3} z_{1}+p_{4} z_{2}+p_{5} z_{1}^{2}+p_{6} z_{1}^{3}\right\}+\xi$ (1)

where $Z_{k}=\mathrm{d} x_{k}, k=1,2$, and $\mathrm{d}$ denotes the individual TPS or HR mixture component of the $k$-th substrate, respectively.

Tables 4 and 5 show the parameter estimator values of the model (1) and the corresponding mean square of the error (MSE) for the six samples $M_{1}-M_{6}$ with the TPS and HR characteristics, respectively. 
Table 4. Estimators of the model parameters and the mean square of the error for $Y=$ TPS

\begin{tabular}{lccccccc}
\hline Mixture & $\hat{p}_{1}$ & $\hat{p}_{2}$ & $\hat{p}_{3}$ & $\hat{p}_{4}$ & $\hat{p}_{5}$ & $\hat{p}_{6}$ & MSE \\
\hline$M_{1}$ & 1.005 & 1.205 & $1.298 \mathrm{e}-2$ & $-2.308 \mathrm{e}-3$ & $-7.254 \mathrm{e}-4$ & $8.008 \mathrm{e}-6$ & 2.872 \\
$M_{2}$ & 0.996 & 1.092 & $1.833 \mathrm{e}-2$ & $-1.289 \mathrm{e}-3$ & $-2.861 \mathrm{e}-4$ & $-5.947 \mathrm{e}-7$ & 3.253 \\
$M_{3}$ & 0.992 & 0.718 & $1.037 \mathrm{e}-2$ & $6.549 \mathrm{e}-3$ & $-6.196 \mathrm{e}-4$ & $9.648 \mathrm{e}-6$ & 3.147 \\
$M_{4}$ & 0.996 & 1.092 & $1.834 \mathrm{e}-2$ & $-1.288 \mathrm{e}-3$ & $-2.861 \mathrm{e}-4$ & $-5.947 \mathrm{e}-7$ & 3.253 \\
$M_{5}$ & 1.004 & 0.493 & $-6.566 \mathrm{e}-3$ & $1.378 \mathrm{e}-2$ & $4.878 \mathrm{e}-4$ & $-5.443 \mathrm{e}-6$ & 0.899 \\
$M_{6}$ & 0.998 & 1.214 & -0.110 & $1.126 \mathrm{e}-2$ & $2.968 \mathrm{e}-3$ & $2.047 \mathrm{e}-5$ & 52.71 \\
\hline
\end{tabular}

Table 5. Estimators of the model parameters and the mean square of the error for $Y=\mathrm{HR}$

\begin{tabular}{lccccccc}
\hline Mixture & $\hat{p}_{1}$ & $\hat{p}_{2}$ & $\hat{p}_{3}$ & $\hat{p}_{4}$ & $\hat{p}_{5}$ & $\hat{p}_{6}$ & MSE \\
\hline$M_{1}$ & 1.007 & 1.354 & $1.312 \mathrm{e}-2$ & $-3.917 \mathrm{e}-3$ & $-1.016 \mathrm{e}-3$ & $1.530 \mathrm{e}-5$ & 4.069 \\
$M_{2}$ & 1.002 & 1.106 & $1.509 \mathrm{e}-2$ & $-1.715 \mathrm{e}-3$ & $-1.668 \mathrm{e}-5$ & $-3.681 \mathrm{e}-6$ & 3.051 \\
$M_{3}$ & 0.998 & 0.7463 & $2.623 \mathrm{e}-2$ & $6.815 \mathrm{e}-3$ & $-1.239 \mathrm{e}-3$ & $1.847 \mathrm{e}-5$ & 1.364 \\
$M_{4}$ & 1.005 & $5.744 \mathrm{e}-1$ & $1.893 \mathrm{e}-2$ & $1.338 \mathrm{e}-2$ & $-2.936 \mathrm{e}-4$ & $5.125 \mathrm{e}-6$ & 5.087 \\
$M_{5}$ & 1.001 & $4.061 \mathrm{e}-1$ & $1.294 \mathrm{e}-2$ & $2.099 \mathrm{e}-2$ & $-1.521 \mathrm{e}-5$ & $-3.200 \mathrm{e}-7$ & 0.605 \\
$M_{6}$ & $9.548 \mathrm{e}-1$ & 1.184 & $-1.674 \mathrm{e}-3$ & $-2.002 \mathrm{e}-3$ & $-5.358 \mathrm{e}-4$ & $5.536 \mathrm{e}-6$ & 66.074 \\
\hline
\end{tabular}

As shown in Tables 8 and 9, the mixture M6 has the poorest fit. However, note that the functional form of a particular physical characteristic modelled must be the same for all mixtures.
As an example, we include in Figure 1 the graphs of the estimated model and the experimental points for the TPS characteristic of mixture $M_{1}$

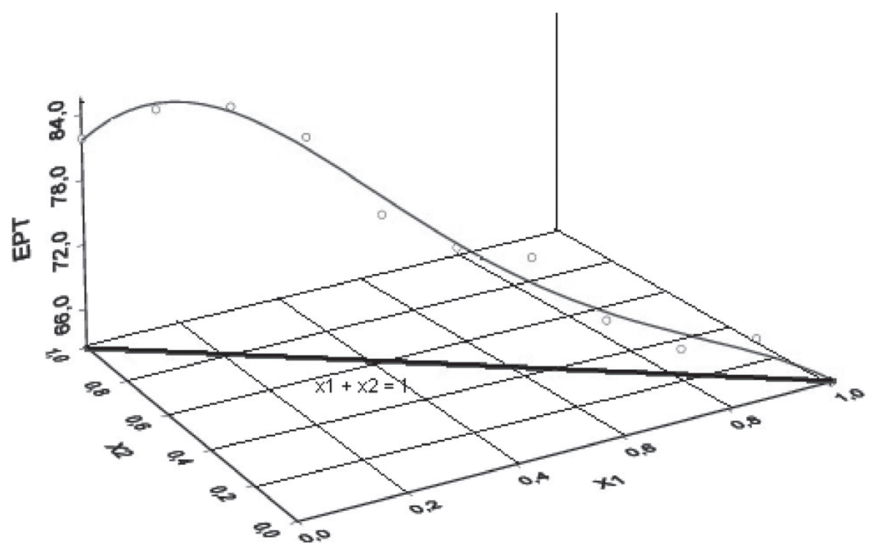

Figure 1. Graphs of the estimated model and the observed TPS data for the mixture $M_{1}$. 


\section{Formulation of mixtures by nonlinear mathematical programming}

We consider the hypothesis proposed by Zamora Morales et al. (2005) as the basis for our formulation of the nonlinear mathematical programming problem. Since the mixture would essentially be used as a support, where the plant receives the nutrients via nutritious solutions, we only consider the physical properties as the design variables, except the variable $\mathrm{OM}$, which is an important representative of the substrate's chemical properties and is therefore incorporated into the general model. In addition, we wish to achieve optimum control of the chemical properties of the nutritious solutions.

Taking these considerations into account, we study the following design variables: the balance of volumes, OM, TPS and HR. The minimising objective function is the cost of the mixture, which is studied via linear functions of the substrate proportions in the mixtures and their individual costs. A basic problem type takes the form (2), where $C_{k}$ is the cost in dollars per litre of the $k$-th substrate (perlite: 0.073 , tezontle: 0.218 , peat: 0.21 and coir: 0.106$) ; x_{k}$ denotes the proportion in litres of the $k$-th substrate; $m_{k}$ is the OM percentage per litre of the $k$-th substrate; $e_{k}$ is the TPS percentage per litre of the $k$-th substrate and $C_{k}$ is the HR percentage per litre of the $k$-th substrate. Now, $O M_{s}$ and $O M_{i}$ are limits or levels of the upper and lower restrictions for OM, respectively. In this study, we consider the limits (0-45) and (46-90); similarly, we define the upper and lower limits for TPS and HR, establishing the following two levels: (55-70) and (71-86) for TPS, and (45-60) and (61-76) for HR. These intervals or levels of restriction, for each of the variables OM, TPS and HR, were established following the recommendations of Abad et al. (1988), for substrates used in a soil-less culture and considering labeling specifications for commercial sustrates. Finally, the respective $\hat{p}_{j}^{k}$ were obtained from Table 5.

$$
\begin{gathered}
\min _{x_{1}, x_{2}} c_{1} x_{1}+c_{2} x_{2} \\
\text { Subject to } \\
x_{1}+x_{2}=1 \\
m_{1} x_{1}+m_{2} x_{2} \leq O M_{s} \\
m_{1} x_{1}+m_{2} x_{2} \geq O M_{i} \\
\hat{p}_{1}^{1} e_{1} x_{1}+\hat{p}_{2}^{1} e_{2} x_{2} \exp \left\{\hat{p}_{3}^{1} e_{1} x_{1}+\hat{p}_{4}^{1} e_{2} x_{2}+\hat{p}_{5}^{1} e_{1} x_{1}^{2}+\hat{p}_{6}^{1} e_{1} x_{1}^{3}\right\} \leq T P S_{s} \\
\hat{p}_{1}^{1} e_{1} x_{1}+\hat{p}_{2}^{1} e_{2} x_{2} \exp \left\{\hat{p}_{3}^{1} e_{1} x_{1}+\hat{p}_{4}^{1} e_{2} x_{2}+\hat{p}_{5}^{1} e_{1} x_{1}^{2}+\hat{p}_{6}^{1} e_{1} x_{1}^{3}\right\} \geq T P S_{i} \\
\hat{p}_{1}^{2} c_{1} x_{1}+\hat{p}_{2}^{2} c_{2} x_{2} \exp \left\{\hat{p}_{3}^{2} c_{1} x_{1}+\hat{p}_{4}^{2} c_{2} x_{2}+\hat{p}_{5}^{2} c_{1} x_{1}^{2}+\hat{p}_{6}^{2} c_{1} x_{1}^{3}\right\} \leq H R_{s} \\
\hat{p}_{1}^{1} e_{1} x_{1}+\hat{p}_{2}^{1} e_{2} x_{2} \exp \left\{\hat{p}_{3}^{1} e_{1} x_{1}+\hat{p}_{4}^{1} e_{2} x_{2}+\hat{p}_{5}^{1} e_{1} x_{1}^{2}+\hat{p}_{6}^{1} e_{1} x_{1}^{3}\right\} \geq H R_{i} \\
0 \leq x_{1} \leq 1,0 \leq x_{2} \leq 1,
\end{gathered}
$$


Potentially, if we combined the four substrates in the two-substrate mixtures ${ }_{4} C_{2}$ with all the possible combinations of the two upper and lower levels for OM, TPS and $\operatorname{HR}\left(2^{3}\right)$ we would have $48=6 \times 8$ possible mixtures. However, Table 6 shows only the mixtures selected by linear or nonlinear mathematical programming. In other words, mixtures for which neither a linear nor a nonlinear optimisation program present a feasible region (i.e. the corresponding optimisation program has no solution) have been removed from Table 10, leaving only the substrate mixtures for soil-less culture with a feasible solution, as either a linear or a nonlinear optimisation program. In cases where the mixture of substrates has no feasible solution in the linear or nonlinear optimisation program, this is noted as NF (there is no feasible solution to the optimisation problem). In addition, the restrictions to the coded variables OM, TPS and HR are coded by assigning "1" to the range with the smaller values and "2" to the higher range.

The results were computed using the commercial software Hyper LINGO/PC, release 6.0 (Winston, 1995). The default optimisation method used by LINGO to solve the nonlinear mathematical programming is that of Generalised Reduced Gradient (GRG) (Bazaraa et al. 2006). As can be seen, the linear programming reduces the total of mixtures by $66.66 \%$, while the nonlinear mathematical programming reduces them by $68.75 \%$; thus, both methods reduce the number of mixtures by very similar amounts. However, note the following two aspects:

Table 6. Mixtures of substrates via nonlinear mathematical programming

\begin{tabular}{|c|c|c|c|c|c|c|}
\hline \multicolumn{3}{|c|}{ Restriction level } & \multicolumn{2}{|c|}{$\begin{array}{c}\text { Solution with } \\
\text { Linear programming }\end{array}$} & \multicolumn{2}{|c|}{$\begin{array}{c}\text { Solution with } \\
\text { Nonlinear programming }\end{array}$} \\
\hline \multirow[t]{2}{*}{$\mathrm{OM}$} & TPS & HR & $x_{1}$ & $x_{2}$ & $x_{1}$ & $x_{2}$ \\
\hline & & & $\%$ & $\%$ & $\%$ & $\%$ \\
\hline \multicolumn{7}{|c|}{ Perlite-coir } \\
\hline 1 & 1 & 1 & 100 & 0 & 100 & 0 \\
\hline 1 & 1 & 2 & 80.2 & 19.8 & 94.0 & 6.0 \\
\hline 1 & 2 & 2 & 55.7 & 44.3 & 57.8 & 42.2 \\
\hline 2 & 2 & 2 & 48.8 & 51.2 & 48.8 & 51.2 \\
\hline \multicolumn{7}{|c|}{ Coir-tezontle } \\
\hline 1 & 1 & 1 & 49.6 & 50.4 & 48.9 & 51.1 \\
\hline 2 & 1 & 2 & 62.0 & 38.0 & 73.5 & 26.5 \\
\hline 2 & 2 & 2 & 96.1 & 3.9 & 96.3 & 3.7 \\
\hline \multicolumn{7}{|c|}{ Perlite-peat } \\
\hline 1 & 1 & 1 & 100 & 0 & 100 & 0 \\
\hline 1 & 1 & 2 & \multicolumn{2}{|c|}{ NF } & 88.2 & 11.8 \\
\hline 1 & 2 & 2 & \multicolumn{2}{|c|}{$\mathrm{NF}$} & 71.9 & 28.1 \\
\hline 2 & 1 & 2 & 44.8 & 55.2 & \multicolumn{2}{|c|}{$\mathrm{NF}$} \\
\hline 2 & 2 & 2 & 15.7 & 84.3 & 48.9 & 51.1 \\
\hline
\end{tabular}


Continued...

\begin{tabular}{|c|c|c|c|c|c|c|}
\hline \multicolumn{3}{|c|}{ Restriction level } & \multicolumn{2}{|c|}{$\begin{array}{c}\text { Solution with } \\
\text { Linear programming }\end{array}$} & \multicolumn{2}{|c|}{$\begin{array}{c}\text { Solution with } \\
\text { Nonlinear programming }\end{array}$} \\
\hline \multirow[t]{2}{*}{$\mathrm{OM}$} & TPS & HR & $x_{1}$ & $x_{2}$ & $x_{1}$ & $x_{2}$ \\
\hline & & & $\%$ & $\%$ & $\%$ & $\%$ \\
\hline \multicolumn{7}{|c|}{ Perlite-tezontle } \\
\hline 1 & 1 & 1 & 100 & 0 & \multicolumn{2}{|c|}{$\mathrm{NF}$} \\
\hline 2 & 1 & 1 & 100 & 0 & \multicolumn{2}{|c|}{$\mathrm{NF}$} \\
\hline \multicolumn{7}{|c|}{ Peat-coir } \\
\hline 2 & 2 & 2 & 10.3 & 89.7 & 0 & 100 \\
\hline \multicolumn{7}{|c|}{ Peat-tezontle } \\
\hline 1 & 1 & 1 & 50 & 50 & 47.9 & 52.1 \\
\hline 1 & 1 & 2 & \multicolumn{2}{|c|}{$\mathrm{NF}$} & 50 & 50 \\
\hline 2 & 1 & 1 & 80 & 20 & \multicolumn{2}{|c|}{$\mathrm{NF}$} \\
\hline 2 & 1 & 2 & 87.9 & 12.1 & 69.7 & 30.3 \\
\hline 2 & 2 & 2 & 100 & 0 & \multicolumn{2}{|c|}{ NF } \\
\hline
\end{tabular}

a) The mixture substrate proportions assigned by the two techniques can vary significantly, as can be seen, for example, in peat-tezontle with restriction levels 212 .

b) Linear mathematical programming is a useful and widely used tool in mixture problems, however, in general the nonlinear programming model achieves a better mixture formulation. But, if a user insists in using a linear solution, he/she could face two situations: One, is that the optimal linear solution might not be feasible under the nonlinear model, and second, that the optimal linear solution might be suboptimal with the respect to the nonlinear solution. Thus, from a practical point of view, the interpretation of these results would be that on occasions non-feasible substrate mixtures used in a soil-less culture are tested or produced, which results in a waste of research time and/or money. Worse still, when linear programming leads us to reject a mixture that would have been accepted by the nonlinear method, we might be rejecting the best possible mixture.

\section{Conclusions}

The aim of this study is to propose a general methodology which can be applied to various situations, with only simple modifications. For example, a mixture formulation in the absence of nutritive substances, or one comprised of substrates that are rich in nutrients and artificial nutrition, could be studied by modifying nonlinear mathematical programming, through restrictions on chemical properties and/or via alternative objective functions.

Alternatively, the presence in the mixture of certain undesirable (desirable) ions or cations could be minimised (maximised). This methodology could be extended to the simultaneous optimisation of several objective functions; for example, to minimise the cost and to maximise the amount of nitrogen in a mixture, which implies the use of nonlinear multiobjective optimisation methods (Steuer, 1986).

Note that the procedure described in Section 3 can be used for modelling various kinds of physical and chemical properties of the mixtures. In addition, the 
nonlinear mathematical programming approach can be adapted by adding new restrictions and/or objective functions for a particular characteristic.

Finally, note that there are few experimental points and a large number of parameters. Nevertheless, on the one hand, we have a sample of equallyspaced experimental points throughout the domain of the variables $x_{1}$ and $x_{2}$ (i.e. the line $x_{1}+x_{2}=1$ ), and the mathematical behaviour considered is a smoother of these variables. Furthermore, our aim is to obtain the best mathematical approximation possible to these points and thus obtain the best results.

A potential user of this methodology (e.g. the commercial producer of a protected crop) could consider using a greater number of experimental points, taking into account the higher initial investment that would be required.

\section{Acknowledgement}

The authors wish to express their gratitude to the editor and the referees for their helpful comments and suggestions. This work was partially supported by the research project CONACYT-45974-F, Mexico.

\section{References}

Abad Berjón, M. and Noguera Murray, P. 1998. Sustrato para el cultivo sin suelo y feriirrigación, Chapter 8. In, Fertirrigación, Cultivos hortícolas y ornamentales, Ed. Cadahia López, C. Ediciones Mundi-Orensa, Bacelona.

Arthanari, T. S., Dodge Y. 1981. Mathematical Programming in Statistics. John Wiley \& Sons, New York.

Bazaraa, M. S., Sherali, H. D., Shetty, C. M. 2006. Nonlinear Programming: Theory and Algorithms, 3rd Edition. Wiley-Interscience.
Burés, S. (1997). Sustratos. Agrotecnia. Madrid, España. $339 \mathrm{pp}$.

Burés S., Martínez, F. X., Llorca, M. 1988. Preliminary study of the application of parametric linear programming in formulation of substrate mixtures. Acta Horticulturae 221, 141-152.

Foth, H.D., Ellis, B.G. 1988. Soil fertility. John Wiley \& S. USA.

Hablin, J. L., Beaton, J. D., Tisdale, S. L., Nelson, W. 1999. Soil fertility and fertilizers. An introduction to nutrient management. Prentice Hall, New Jersey. USA

Gabriel, R., Keirsbulk, W., Engels, H. 1993. A rapid method for the determination of physical properties of growing media. Acta Horticulturae 342, 243-248.

Graybill, F. A., Iyer, H. K. 1994. Regression Analysis. Duxbury Pr. USA.

Lam, L. (2011). An introduction to S-PLUS for Windows. http://www.splusbook.com/

Pire, R., Pereira, A. 2003. Propiedades físicas de componentes de sustratos de uso común en la horticultura del estado de Lara, Venezuela. Propuesta metodológica. Bioagro. ISSN 1316-1361.

North Carolina State University, 2007. Horticultural substrates laboratory. http://www2.ncsu.edu/unity/ lockers/project/hortsublab/ diagnostic/index. html

Rao, S. S. 1979. Optimization Theory and Applications. Wiley Eastern Limited.

Resh, H.M. 2002a. Hydroponic food production. Newconcept Press Inc. New Jersey. USA.

Resh, H.M. 2002b. Hydroponics. Questions and answers for successful growing. Newconcept Press Inc. New Jersey. USA.

Russell, E.J. 2008. Soil conditions and plant growth. Biotech books. USA. 
Steuer, R. E. 1986. Multiple criteria optimization: Theory, computation and applications. John Wiley, New York.

Winston, W. L. 1995. Introduction to mathematical programming: Applications and algorithms. Duxbury Press.
Zamora Morales, B. P., Galvis Spínola, A., Volke Haller, V. H., Sánchez García, P., Espinosa Victoria, D. 2005. Formulación de mezclas de sustratos mediante programación lineal. Interciencia: Revista de ciencia y tecnología de América, ISSN 0378-1844, 30(6), 365-369. 
